\title{
Jewellery Investment Casting Machines
}

\author{
Peter E. Gainsbury \\ The Worshipful Company of Goldsmiths, London
}

Investment casting is by far the most widely used technique for the production of gold jewellery. Each year, large quantities of carat gold alloys are processed by this method. It is therefore of importance that machines used for jewellery investment casting should meet the efficiency and quality standards expected in large scale manufacturing equipment.

Despite the extensive use of the related lost wax process in antiquity, the modern technique of investment casting has been applied in industry for a relatively short period. Its use in the casting of dental restorations in 1907 was not followed immediately by other applications. Thus, it was not until the late 1930 's that it was taken up by manufacturing jewellers and it was only during World World II that experience of it as an engineering process was built up. Curiously, however, the process as used in jewellery production today owes little to engineering investment casting and the techniques used in the two areas have developed almost independently.

At the present time, jewellery casting is passing through an era of considerable change; it appears likely that the period of empirical development of materials and equipment of the past thirty years is coming to an end and that a process will emerge with a better tehnical basis than has been the case to date.

\section{The Basic Process}

The starting point for the production of a jewellery casting is a master pattern of the part, normally made of metal to the highest standard of detail and finish. From the pattern, a negative die is made to be used for the production of expendable patterns. For this, the most usual procedure is to vulcanize a solid block of rubber around the master using heat and pressure, subsequently parting the die and removing it by judicious cutting with a surgical scalpel. Two-part dies made in this way can reproduce complex designs with heavy undercuts by low pressure injection of molten wax, the flexible rubber permitting the patterns to be removed without distortion. Other die materials used are cast elastomers, cast epoxy resins and low fusing alloys. Rigid or metal dies may be used with higher wax injection pressures than flexible materials to obtain the finest detail and accuracy. If large production runs are called for, patterns may be produced in metal dies by the injection moulding of plastics.
Wax patterns are set up in large or small numbers depending on production requirements and casting machine capacity. Various techniques are used for setting up but the most common for quantity production is to attach the patterns radially by short moulded-on sprues to a heavy central feeder (Figure 1).

Once set up, the patterns are placed in a mould and embedded in investment. Almost all jewellery investments are based on combinations of plaster and silica which are mixed to fluid slurries with water and formulated to set in about 10 minutes from the com-

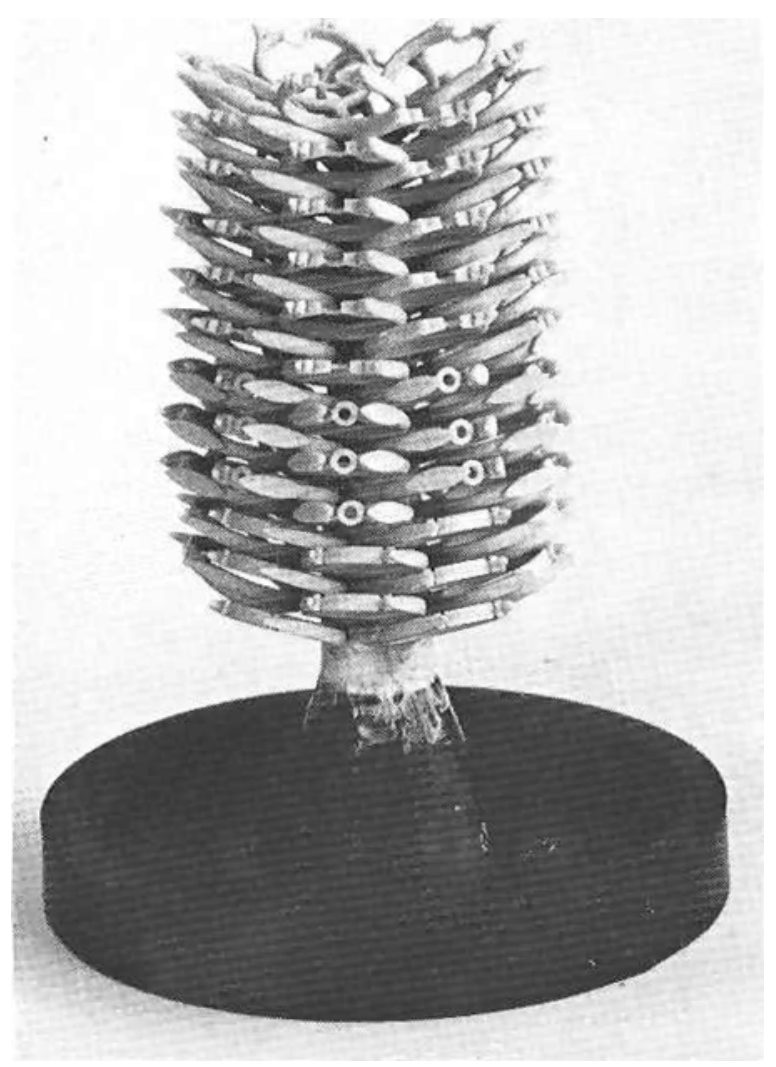

Fig. I This wax tree has been built by attaching individually made ring patterns to the central feeder. The tree is now ready for investing 
Fig. 2 Simple spring-driven centrifugal casting machine. The crucible for torch melting and an empty investment flask are seen in position on the articulated casting arm. An adjustable counterweight is fitted at the opposite extremity of the arm

Photograph by courtesy of Hoben Davis Ltd., Newcastle u. Lyme, Staffs., England

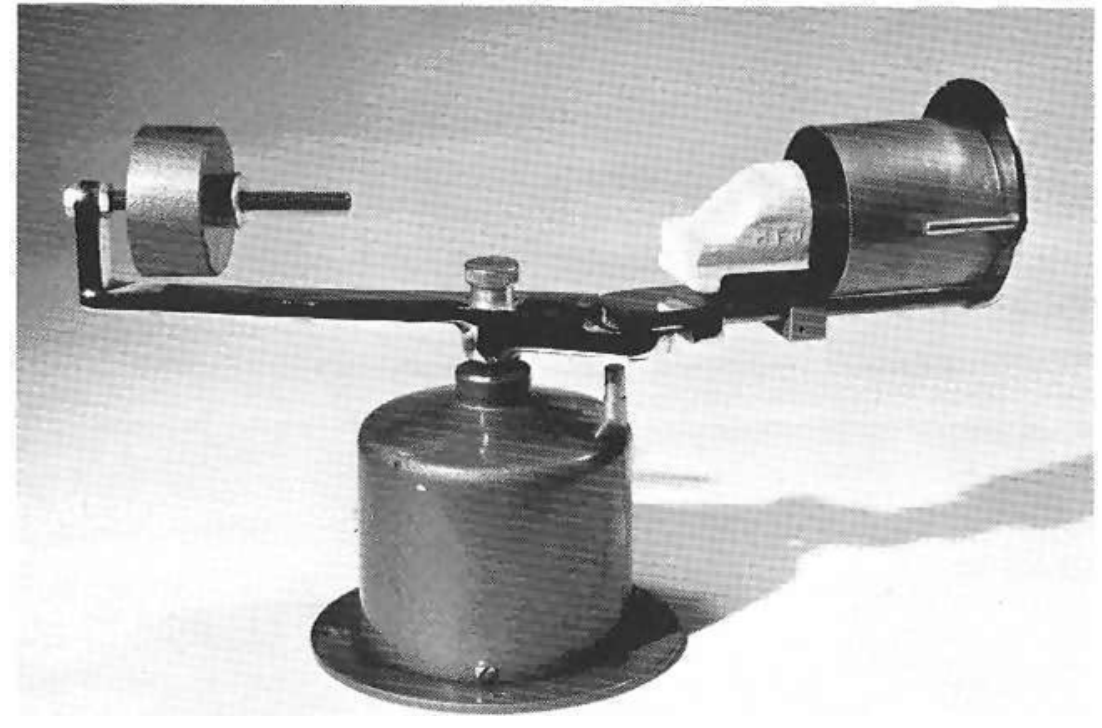

mencement of mixing. Solid block moulds are the general rule, multiple investing procedures with inner and backing investments are not used in jewellery casting practice. Most investment moulds are formed in cylindrical heat-resisting metal flasks. Air entrapped on the surface of patterns is removed before setting commences by subjecting the mould to a vacuum sufficient to boil the water in the mix.

Set moulds are dewaxed by low temperature heating, either in air or steam, and then fired to between 700 and $800^{\circ} \mathrm{C}$ to cure the investment and burn out carbonaceous residues. Before casting, jewellery moulds are normally cooled to between 300 and $700^{\circ} \mathrm{C}$ depending on the casting temperature of the alloy being used and the nature of the patterns in the mould.

\section{Casting Machines}

Jewellery-type investment moulds cannot be filled by simple gravity pouring. The combination of unvented moulds with low permeability refractory, the need to reproduce fine detail and delicate sections together with the relatively small size of melts and the consequent low hydrostatic head and low thermal content of the metal preclude this possibility. Casting of the metal into the mould is therefore almost invariably carried out in some form of casting machine.

A basic function of the casting machine is to apply pressure to the molten metal so that it penetrates and fills the mould completely. This same pressure may also be used to effect transfer of the molten metal to the mould from the crucible when this is part of the machine. Centrifugal force, pressure or vacuum, or a combination of them, are used to perform these two functions. The machine may also have built into it a means of melting the metal or a crucible or hearth in which the metal can be melted by external torch heating. Ancillary functions provided on the more sophisticated machines are melt temperature indication and regulation, atmosphere control and casting pressure regulation.

\section{Centrifugal Casting Machines}

'Centrifugal' machines for casting jewellery are not truly centrifugal, the mould does not rotate on its own axis but is fitted at one extremity of a balanced arm, the rotation of which develops the necessary pressure to force the metal into the mould (Figure 2).

Such casting machines were initially spring-driven but modern machines are almost invariably powerdriven at speeds up to around $300 \mathrm{r} / \mathrm{min}$ and the most satisfactory types have rotation speed or torque regulators. Power-driven rotation provides constant speed and ensures that the centripetal force continues to be applied until the metal has solidified. Also, the possibility of run-back is eliminated and, in complete contrast to spring-driven machines, a slow take-off speed can be followed by an increase in speed to reduce the possibility of turbulence and allow maximum consolidation of the metal in the mould. Springdriven rotation would in any case be impractical for large capacity machines which may be able to cast up to $7 \mathrm{~kg}$ of 18 carat gold alloy.

Torch, electrical resistance and high frequency induction melting are all used on centrifugal casting machines. There are a few large capacity machines in which the metal is melted in a conventional crucible in a separate furnace, the crucible with its molten charge being transferred to a carrying cradle on the machine immediately before the release of the casting arm. However, direct melting on the machine is by far the most common practice. 


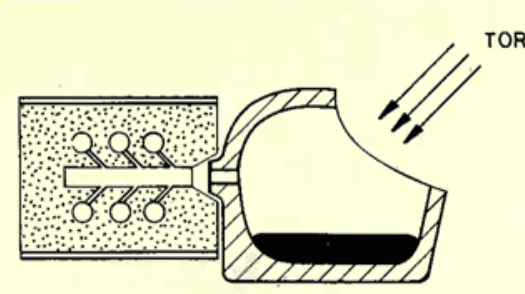

MELTING

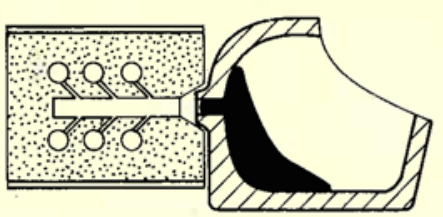

COMMENCEMENT OF ROTATION

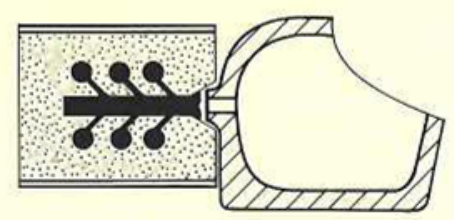

MOULD FILLED

Fig. 3 Schematic representation of casting in a torch melting centrifugal machine such as that of Figure 2 . The axis of rotation is to the right of the illustration

\section{Torch Melting Machines}

Torch melting is carried out in shallow hearth-type crucibles which are hooded at the front end where there is a central hole through which the metal is transferred to the investment mould (Figure 3). The crucibles for torch melting are normally moulded in high-alumina fireclays and, if protected from mechanical damage, have good lives. Considerable thought has gone into their design in recent years to ensure efficient melting, smooth transfer of the melt to the mould and avoidance of tangential losses during the acceleration of the casting machine arm. While a shallow configuration of the hearth of the crucible provides for rapid melting, it may also promote oxidation of the charge or gas absorption as a result of the large exposed surface of molten metal. However, skilled melters using an efficient torch have little difficulty in producing gas- and oxide-free melts of any normal jewellery gold alloy. Town gas, natural gas, propane or acetylene are used as torch fuels with compressed air or oxygen as supporters. Preferences are dictated by availability but combinations which could lead to overheating of the melt are to be avoided.

Torch melting suffers from the great technical disadvantage that temperature control and protection of the melt against oxidation are not possible. The preparation of the metal in optimum condition for casting is dependant on the skill of the operator and the use of refined melting aids such as vacuum or inert atmosphere is precluded.

It is unlikely that any major development can be expected in torch melting centrifugal casting. No doubt, improvement of the details of torch and machine design will continue but it must be concluded that this process is being superseded. Although it remains a very adequate production method, particularly for small-scale work, it would appear inevitable that for jewellery of the highest quality it must give way to techniques which permit better control of the condition of the melt.

\section{Resistance Melting Machines}

The use of resistance melting has been confined principally to only two types of casting machines.

One machine utilizes a horizontal, cylindrical, wirewound resistance furnace. A cylindrical graphite crucible is used with a charging hole at one end and a casting hole at the other. The furnace is rigidly mounted on a straight casting arm and the mould is clamped against the casting end of the furnace by a stepped sliding plate. A thermocouple fitted in a groove in the outer crucible wall, indicates and controls the furnace temperature. The enclosed design of the graphite crucible ensures clean gas-free melts of alloys melting at up to around $1000^{\circ} \mathrm{C}$. The machine, however, has limited capacity with a maximum of about $620 \mathrm{~g}$ of 18 carat gold alloy. Moreover, melting is slow, it is difficult to observe the melt condition and the arm is spring-driven.

A resistance melting machine of greater current interest is produced in a range of sizes with maximum 18 carat gold capacities of 200 to $2000 \mathrm{~g}$. Originally designed for dental applications, the system is unique in that carbon resistance heating is employed together with metal transfer by tilting in conjunction with the centrifugal configuration. The furnace is of tubular form with the axis vertical for melting and is rotated on trunnions to the horizontal position for casting. It is mounted on the edge of a power-driven horizontal turntable which carries a counterweight or a second furnace on the opposite edge. Initially, the mould is placed mouth downwards over the top of the melting crucible and clamped in position. When the metal is melted and the turntable rotated, a trip releases the furnace and it tilts to the horizontal position with the mould outwards (Figure 4). Tilting is controlled by an oil dash-pot, so that smoother transfer of the metal to the mould is obtained than with other systems.

The metal may be melted in a graphite or ceramic crucible fitted inside a ceramic liner to the tubular resistance element. There is no practical limit to alloy melting temperature as far as jewellery alloys are con- 
Fig. 4 Schematic representation of casting in a carbon resistance melting centrifugal machine. Metal pouring is achieved by progressive tilting of the mouldfurnace assembly to the horizontal position at the commencement of rotation: The rotation axis is to the right of the illustration. After documents from Arno Lindner Fabrik, Munich, West Germany

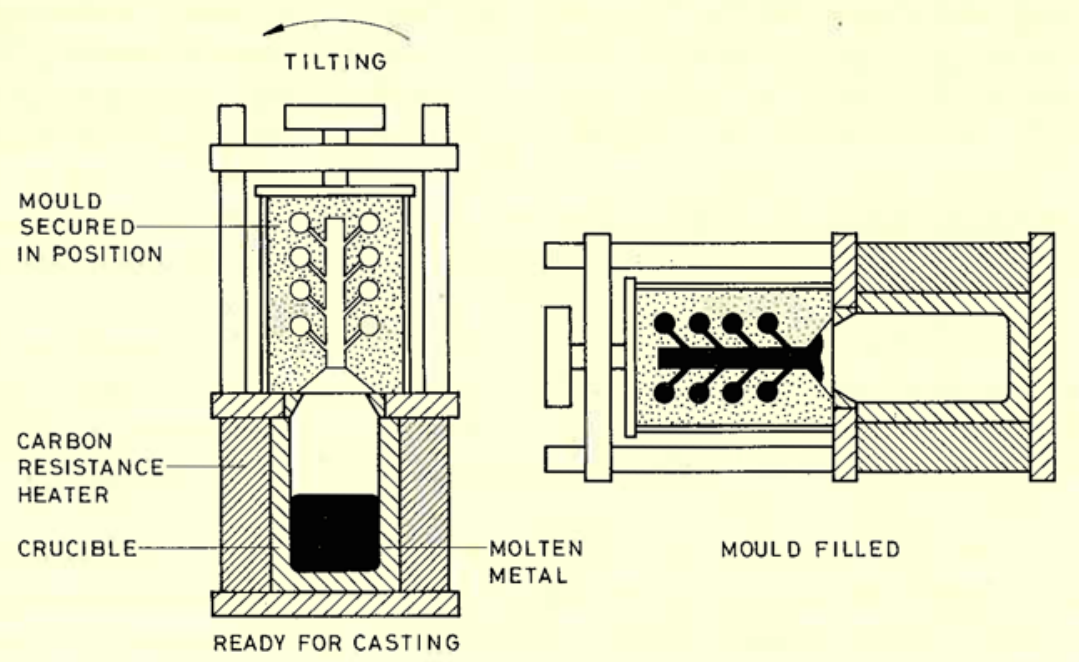

cerned. Melting conditions are inevitably reducing and very satisfactory for normal coloured gold alloys. With white gold alloys, however, particularly the high melting range soft alloys, there is a danger of pick-up of deleterious impurities such as sulphur from the graphite or silicon from the ceramic, leading to serious hot shortness and apparent embrittlement. Furnace temperature may be controlled by a thermocouple positioned between the resistance element and the furnace liner but with this configuration there may be a considerable difference between the indicated and the actual metal temperatures.

In some respects, the system described above embodies the most satisfactory application of the centrifugal principle to jewellery casting. Melting conditions are excellent, some control of the melt temperature is possible, smooth, controlled metal transfer is obtained and the cost of the equipment is modest. Disadvantages are the limited maximum capacity, the impossibility of loading bulky scrap, slow melting, the fact that observation of the melt immediately prior to casting is not practical and limited heating element life. While resistance melting lends itself more readily to smooth centrifugal metal transfer than induction melting (as will be seen below), it can be predicted that this advantage will not be relevant in future if, as is likely, static casting processes become pre-eminent.

\section{High Frequency Induction Melting Machines}

At the present time, induction melting centrifugal casting machines are probably the most widely produced of all types for jewellery casting. They are marketed by some ten or more manufacturers in Europe and the U.S.A.

Basically, all these machines are very similar with integral water-cooled valve or solid state generators of
3 to $18 \mathrm{kVA}$ nominal output through short retractable coils. Melting is in vertical crucibles generally mounted on a rigid counter-balanced power-driven centrifugal casting arm rotating in the horizontal plane. Melting capacities range from $150 \mathrm{~g}$ to $5 \mathrm{~kg}$ of 18 carat gold alloy.

It is considered that the weak point of most such machines is the mechanism of metal transfer from the crucible to the mould. This depends on the fact that the crucible walls slope outwards a few degrees from the vertical and a pouring hole is provided at the top of the side facing outwards from the centre of rotation and aligned with the centre of the mouth of the horizontal mould. With ceramic crucibles the half of the crucible on the pouring hole side may be covered with an integrally moulded hood, but with the more commonly used graphite crucibles the pouring hole is

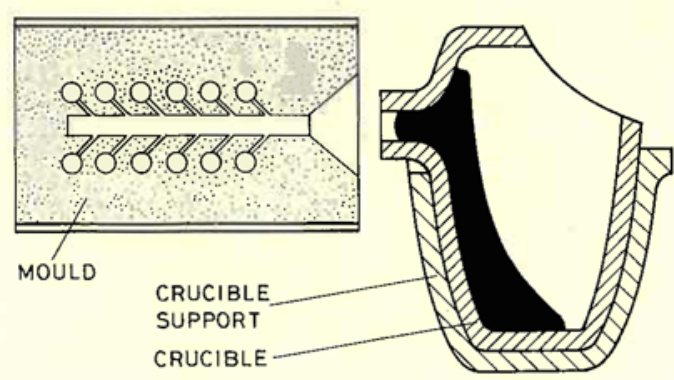

Fig. 5 Schematic representation of metal transfer in an induction melting centrifugal casting machine. Once the required melt temperature has been reached, the induction coil is retracted and rotation is triggered. Upon attainment of sufficient centripetal force, the metal is projected through the opening in the crucible wall and into the mould cavity. The rotation axis is to the right of the illustration 
a semi-circular depression in the top rim, which is covered with a loose flat lid. When the centrifugal arm is set in motion, the centripetal force causes the molten metal to climb up the inclined crucible wall opposite to the centre of rotation until it reaches the pouring hole through which it is rapidly flung into the mould (Figure 5). It is evident that only a small component of the centripetal force is available to cause the metal to climb the crucible wall but that as soon as the metal reaches the pouring hole the full force is suddenly applied to it and it is very rapidly and turbulently thrown into the mould. If the rotation speed and thus the centripetal force is high, then excessive entrainment of air in the metal stream may result. This effect can cause considerable and sometimes very puzzling porosity in castings. If air is entrapped in the metal and solidification is rapid, as it normally is, there may not be time for the air to be forced out of the mould cavity before an initial solid metal skin forms on the mould walls. With the slower solidification of metal within the interior of the casting, the centrifugal action causes air bubbles to be trapped below the surface of castings at points towards the axis of rotation. It is therefore essential that some form of speed control which permits the progressive application of centrifugal force be fitted onto machines of the above design.

When temperature control is fitted to induction melting machines it may be by use of radiation pyrometers or immersion thermocouples. Pyrometer

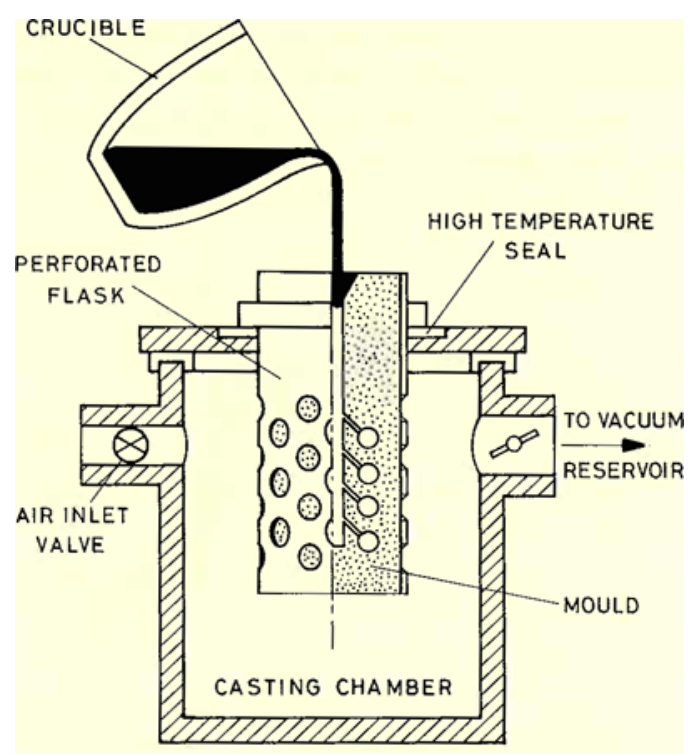

Fig. 6 Principle of vacuum assisted static investment casting with a perforated flask. As the metal is poured from the crucible, vacuum from the reservoir is applied to the casting chamber and is transmitted to the mould cavity via the perforations in the flask and the pores in the investment readings can be affected by oxide films at the surface of the melt, by fumes or by inefficient stirring in radio frequency melting. Thermocouples can be affected by stray currents and sheath life is often too short. Thus, both control methods have limitations but optical pyrometry is much simpler for use with a crucible which has to move and to which it is therefore difficult to arrange electrical connections.

Melting of coloured gold alloys is normally in machined graphite or electrically conducting silicon carbide crucibles. With the very high frequency currents employed, coupling to the metal charge is not too efficient and more effective melting is obtained by the use of the crucible as a susceptor. The use of graphite crucibles also provides some measure of protective atmosphere over the melt, though for alloys of high base metal content this is insufficient to prevent oxidation and most machines have facilities for introducing an inert or reducing atmosphere above the crucible. Atmospheres used have included natural gas, nitrogen-hydrogen mixtures and argon. With the latter gas, difficulties can arise if it contains moisture. Reduction of water vapour by the hot graphite crucible can occur, resulting in hydrogen absorption into the melt and subsequent gas porosity in the castings.

\section{Static Casting Machines}

Simple air- or steam-pressure casting machines have been in use in parallel with centrifugal machines in dental casting for far longer than jewellery casting has been a commercial proposition. These machines were only suitable for casting small melts made in the top of the mould itself and early commercial jewellery casting was primarily with centrifugal machines. For manufacture of jewellery on a small scale, simple vacuum assisted machines began to appear some years ago in the United States and limited use was made of larger equipment of the same general type for heavy section silverware casting. The principle of all these machines was that the mould was placed on a heatresisting gasket on a flat table over a hole connected to a vacuum pump and vacuum was applied to the base of the mould while the molten metal was poured. The low pressure in the mould cavity caused atmospheric pressure to force the metal into the mould, filling being assisted by reduction of the cushion effect of the air in the mould cavity.

These techniques though effective for small moulds or heavy section casting are not suitable for production-scale jewellery casting and it was not until 1970 that commercial equipment for static investment casting became available to manufacturers. The process, correctly described as a 'vacuum assisted casting' and not as 'vacuum casting', is simple. A perforated moulding flask is used which is fitted with a heavy flange on the upper or molten metal entry end. 
Moulds are invested in the usual way with a single sprue entry to the cavity. The machine (Figure 6) consists of a cylindrical casting chamber, large enough to accommodate the largest mould used, and which has an open top with a flange corresponding to the flange on the flask. The casting chamber is fitted inside a vacuum chamber of large volume and is isolated from it by a large diameter quick-opening valve. The vacuum chamber is provided with a simple gauge and is evacuated by a rotary pump. The metal is melted independently in any suitable crucible furnace and when casting is to be carried out, the vacuum chamber is pumped out with the valve closed and the hot investment mould is seated on the flange of the casting chamber with a heat-resisting gasket interposed between the flanges. The molten metal is simply poured into the mould by hand, the vacuum valve being opened momentarily before the metal stream strikes the mould surface.

The technique is giving excellent service for both the high volume production of small jewellery castings and for large size castings much too heavy for production on centrifugal machines. Its disadvantages are the expense of the specially designed flasks and the difficulty of seating them accurately and removing them from the casting machine at temperatures up to around $700^{\circ} \mathrm{C}$. Several developments have, however, been introduced by equipment manufacturers which aim at simpler and more economical operation.

Although vacuum assisted casting techniques work extremely well in some operators' hands, there is still controversy about their effectiveness for thin section casting. This is because correct sprueing is probably more critical than in centrifugal casting and the time of opening the vacuum valve in relation to pouring the metal is of prime importance. For this reason, automatic machines where the mould cavity is evacuated in advance with the mould in a sealed chamber have been developed. These are probably the most sophisticated casting machines currently available.

The machines (Figure 7) feature a fully enclosed casting chamber. The pouring hole in the chamber lid is sealed to the bottom of the melting furnace, which is fitted with a bottom pouring crucible closed from above by a graphite stopper carrying a thermocouple. Melting in the larger machines is by medium frequency induction. Simple transfer of the molten metal from the crucible to the mould is monitored by the attainment of either the desired temperature of the metal or the partial pressure in the casting chamber. In automatic machines of recent design, the induction coil, crucible and metal release mechanism are completely enclosed in a separate metal vacuum chamber, thus enabling full vacuum or inert atmosphere melting and casting. Machines are

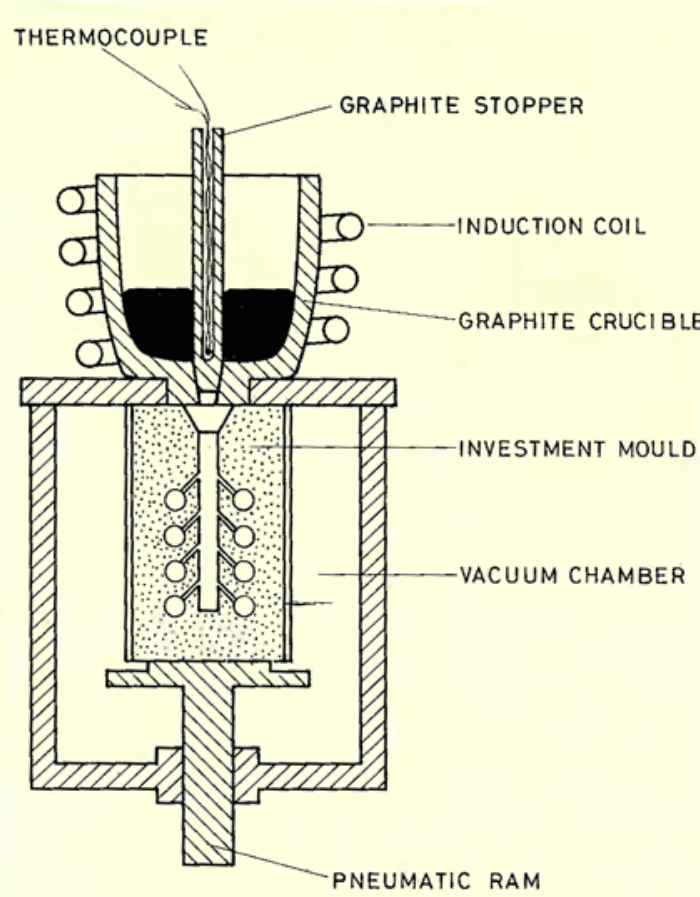

Fig. 7 Schematic representation of the bottom pouring type of vacuum assisted static investment casting machine with induction melting. The casting chamber is evacuated in advance. Upon attainment of casting conditions the graphite stopper rod is lifted and the molten metal fills the mould cavity under the combined effects of gravity and atmospheric pressure

also being developed in which the induction coil and the mould are enclosed in a single vacuum chamber. Pouring is generally by tilting of the crucible via external controls and in the simplest case, casting pressure is established by evacuating the chamber during melting and then exposing to atmospheric pressure at the moment of pouring the metal into the mould. A refinement of this process is to provide a vacuum take-off from the mould support table so that vacuum can be maintained on the base of the mould when atmospheric pressure is admitted to the main casting chamber.

Most lower carat gold alloys contain zinc and should not be melted at low pressures. For such alloys full vacuum chamber machines are used with back filling with an inert gas to a partial pressure sufficient to eliminate volatilization of alloying elements. In this case the possibility of applying full vacuum to the base of the mould at the moment of casting is a decided advantage.

\section{Hybrid Machines}

A development of centrifugal induction melting casting machines has been to enclose the melting coil and casting arm in a vacuum chamber. This move introduced many design problems which inevitably 


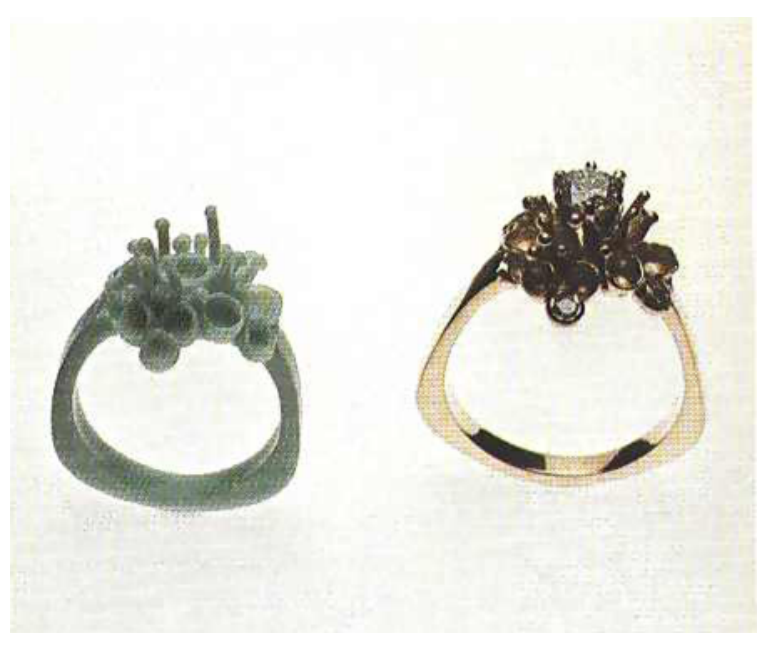

resulted in machines much more expensive than their air melting counterparts with possibly little improvement in the quality of the castings.

Another approach has been the application of a vacuum to the bottom of investment moulds while casting was carried out by normal centrifugal methods. The reasoning is that the vacuum draws 'harmful' gases out of the mould before the metal enters and that atmospheric pressure on the metal helps mould filling. It is unlikely, however, that any 'harmful' gases would be present in properly fired investment moulds. Vacuum is not applied to the base of the mould until rotation of the casting arm commences, thus, no significant purging of the mould occurs before the entry of the metal and in the best of cases only very partial vacuum is achieved before metal solidification. Clearly, a factor in the investment casting process is that air or gas must be forced out of the unvented mould cavity through the low porosity investment material by the pressure of the molten metal. There is ample practical evidence that assistance by either centripetal force or atmospheric pressure and gravity suffices to overcome this problem. The beneficial results, if any, of compounded rotation and inefficient vacuum assistance do not justify the complication and expense of such hybrid machines.

\section{Discussion}

While it is true that centrifugal casting has been the backbone of the development of jewellery casting to its present level of efficiency (Figure 8), it is suggested that persistence in the use of the technique is now more based on habit than on necessity. However, it must be admitted that with the current level of knowledge of the parameters of casting, the powerdriven centrifugal machine with torch melting is still the most economical, versatile and reliable method of casting the whole range of jewellery alloys.
Fig. 8 A wax pattern and a finished 18 carat gold and diamond ring cast from the same pattern Photograph by courtesy of Julie Crossland

The combination of efficient induction melting techniques with centrifugal methods of metal transfer has led to a decrease in the efficiency of the latter and to a loss of some of the advantages of the former. Further, the turbulent transfer of large quantities of expensive metal in the molten state by high speed spinning and climbing of a near-vertical crucible wall appears inefficient, physically and economically hazardous and mechanically expensive: With melts of the average size cast today there is ample time to transfer metal smoothly into moulds by simple tilting of the crucible by the well tried Durville method or perhaps most effectively, but with greater technical difficulty, by bottom pouring. Altogether, it seems a decided advantage to work with gravity, instead of against it, in order to transfer the metal, fill the mould and obtain a sharp impression.

Casting machines of the future should have flexibility allowing large or relatively small melts to be made under vacuum or controlled atmosphere with efficient temperature control. Pouring should be by means allowing smooth entry of the metal stream into the mould and the necessary pressure on the metal should be as uniform as possible throughout the mould and automatically or semi-automatically applied at the correct moment. However, before such ideal machines can be fully developed, further work needs to be done on the many interdependent parameters which affect the efficiency of the casting machine itself, which after all is only one factor in a many facetted overall process.

\section{Acknowledgements}

The writer is indebted to the Worshipful Company of Goldsmiths for permission to publish this article and to his colleague Chris Walton for many stimulating discussions.

Interested readers are invited to contact the author for more information. Mr. Gainsbury is Director of Research, Technical Department, The Worshipful Company of Goldsmiths, Goldsmiths' Hall, Foster Lane, London EC2V 6BN, England. 